\title{
COMBATE DE SONTOL (Cyperus iria L.) EN ARROZ1
}

\author{
Franklin Herrera ${ }^{2}$, Renán Agiiero 3
}

\section{RESUMEN}

Combate de sontol (Cyperus iria L.) en arroz. En la Hacienda arrocera "El Pelón", ubicada en Liberia, Guanacaste, en un lote altamente infestado por Cyperus iria, se realizó este experimento con el propósito de determinar la efectividad en el combate de esta maleza y la selectividad al arroz, de varios herbicidas hormonales y sulfonilureas y estimar preliminarmente el efecto negativo de Cyperus iria en el arroz. Los tratamientos evaluados incluyeron tres nuevas sulfonilureas, seis tratamientos herbicidas de uso recomendado en el control de ciperáceas y dicotiledóneas y un testigo sin control de $C$. iria. La aplicación de los herbicidas se hizo siguiendo las recomendaciones que para cada uno hicieron las casas comerciales. Se utilizó el cultivar de arroz CR 1821, el terreno se mantuvo saturado durante todo el experimento. Los herbicidas más efectivos para el control de $C$. iria, fueron las sulfonilureas, bensulfurón-etil y pyrazosul-furón-metil, a su vez, fueron los tratamientos donde se obtuvo el mayor rendimiento del arroz. Los herbicidas que tradicionalmente se usan para el control de ciperáceas, mostraron porcentajes intermedios de control de esta maleza.

Cuando no se controló el $C$. iria, aproximadamente a los 30 días después de la siembra, logró cubrir totalmente las plantas de arroz, cumplió su ciclo de vida a los 70 días y redujo el rendimiento del arroz en granza en un $50 \%$.

\begin{abstract}
Control of flatsedge (Cyperus iria L.) in rice. This experiment was conducted in a highly $C$. iria infested plot at "El Pelon" rice farm in Liberia, Costa Rica, to determine the control effectiveness of this weed by several hormonal and sulfonylurea herbicides and their selectivity to the rice crop, and to preliminarily estimate the negative effect of $C$. iria on the crop. The evaluated treatments included three new sulfonylureas, six herbicides recommended for cyperaceous and dicotyledonous control and an unweeded control. The herbicides were app!ied following each of the manufacturer's recommendations. The rice cultivar used was CR 1821 and the ground was kept saturated during the whole trial. The sulfonylurea herbicides, bensulfuron-ethyl and pyrazosulfuron-methyl, were the most effective in controlling $C$. iria, and they also were the highest yielding treatments. The herbicides commonly used for the cyperaceous control, showed intermediate control percentages of these weeds.

When $C$. iria was not controlled, at approximately 30 days after planting, it completely covered the rice plants, completed its !ife cycle at 70 days and reduced the rice yield by $50 \%$.
\end{abstract}

\section{INTRODUCCIÓN}

Las ciperáceas, constituyen un componente importante del complejo de malezas que afectan al cultivo del arroz en diferentes partes del mundo, (Holm et al, 1977; Irat, 1978, Soto y Agtiero, 1992). En Costa Rica, prevalecen Cyperus rotundus, Cyperus esculentus y Scleria pterota en los sistemas de siembra de arroz de secano y secano favorecido; mientras que Cyperus iria, Fimbristylis sp y en algunas zonas, Scleria sp. son las más comunes en el sistema anegado, (Soto y Agtiero, 1992; Alpizar y Barrantes, 1994).

En los últimos años, en las áreas de siembra de arroz y especialmente en Guanacaste, se ha observado un aumento en la distribución y densidades de población de $C y$ perus iria, atribuible a posibles fallas en el manejo de los herbicidas, a la falta de integrar otras estrategias de combate y a que se han modificado algunas prácticas de manejo del cultivo.

\footnotetext{
1 Presentado en la XL Reunión Anual del PCCMCA en Costa Rica, América Central. 13 al 19 de marzo, 1994.

2 Mag.Sc. Programa de Malezas. Estación Experimental Fabio Baudrit M., Universidad de Costa Rica.

3 Mag.Sc.. Centro de Investigación en Protección de Cultivos, Universidad de Costa Rica..
} 
Cyperus iria es originaria del trópico Asiático, de ciclo anual y reproducción sexual (cada planta puede producir hasta 5000 semillas viables). Se le menciona entre las tres ciperáceas más importantes en el cultivo del arroz, en el mundo. Prospera bien en suelos húmedos y con buena exposición de luz, por lo que las condiciones en que se siembra arroz anegado le favorecen, sin embargo, se informa de su presencia en 17 cultivos más, (Holm et al, 1977).

La estrategia más común para el combate de esta maleza, ha sido el uso de herbicidas, entre los que destacan el grupo fenoxiacético, sin embargo, presentan la característica de que deben aplicarse en pleno macollamiento del arroz; su uso en estados muy tempranas del arroz y durante la fase reproductiva, causa daños importantes al cultivo (Soto y Agiiero, 1992; Irat, 1978).

Las sulfonilureas es otro grupo reciente que incluye herbicidas efectivos para controlar ciperáceas en arroz, pudiendo aplicarse en estados más tempranas. Dentro de ese grupo, el bensulfurón se ha utilizado en otros países, para el combate de alismatáceas, ciperáceas y malezas de hoja ancha en arroz, (Yagiiey Tylko, 1992).

También se han formulado algunas mezclas de herbicidas hormonales con otros grupos, que se usan con éxito en arroz. Debido a la disponibilidad de varios herbicidas para el control de ciperáceas y a que el problema de las mismas persiste en diferentes grados, se planteó este experimento con los siguientes objetivos:

1. Determinar la efectividad en el combate de Cyperus iria y la selectividad al arroz, de varios herbicidas hormonales y sulfonilureas.

2. Estimar preliminarmente el efecto negativo de Cyperus iria en el arroz.

\section{MATERIALES Y MÉTODOS}

Este experimento se realizó en un área experimental de la hacienda El Pelón, Liberia, Guanacaste. En una "melga" especialmente preparada para la siembra de arroz, se aplicó riego hasta saturar el suelo, luego se sembró semilla de arroz cv. 1821 a la densidad que usa la finca. El manejo del arroz, excepto en lo que respecta a control de malezas se hizo igual que en el resto del cultivo comercial. El campo se mantuvo siempre saturado (pero sin láminade agua superficial). Los tratamientos herbicidas evaluados (Cuadro 1), se hicieron según las recomendaciones técnicas de cada casa comercial.

Cuadro 1. Nombre técnico y dosis de los herbicidas evaluados, momento de aplicación y estado de desarrollo del $C$. iria y el arroz al aplicar cada herbicida. Guanacaste, 1993.

\begin{tabular}{|c|c|c|c|c|}
\hline \multirow[t]{2}{*}{ Herbicida } & \multirow{2}{*}{$\begin{array}{l}\text { Dosis } \\
\mathrm{g} \text { i.a/ha }\end{array}$} & \multicolumn{2}{|c|}{ Estado de Desarrollo } & \multirow{2}{*}{$\begin{array}{c}\text { Aplicación } \\
\text { SDS }{ }^{1 /}\end{array}$} \\
\hline & & C. iria & Arroz & \\
\hline benzulfurón-metil & 48 & 2-3 hojas & 4-5 hojas & 3,5 \\
\hline pyrazosulfurón-etil & 20 & 1-2 hojas & $1-2$ hojas & 2 \\
\hline Hoe 404 (Nombre clave) & 60 (P.C) & 4-5 hojas & macollamiento & 4,5 \\
\hline triclopir & 144 & $1-2$ hojas & 1-2 hojas. & 2 \\
\hline triclopir & 192 & 4-5 hojas & macollamiento & 4,5 \\
\hline bentazón + MCPA & $720+90$ & 4-5 hojas & macollamiento & 4,5 \\
\hline $2,4-\mathrm{D}$ & 500 & 4-5 hojas & macollamiento & 4,5 \\
\hline 2,4-D + MCPA & 480 & 2-3 hojas & 4-5 hojas & 3,5 \\
\hline ioxinil + 2,4-D & $100+600$ & 4-5 hojas & macollamiento & 4,5 \\
\hline
\end{tabular}

" SDS= Semanas después de la siembra del arroz 
En el Cuadro 2 se indican las formulaciones comerciales utilizadas en el experimento.

Cuadro 2. Formulaciones comerciales utilizadas en el experimento "Combate de Sontol en arroz", Guanacaste, 1993."

\begin{tabular}{|c|c|}
\hline $\begin{array}{l}\text { Nombre técnico } \\
\text { del herbicida }\end{array}$ & $\begin{array}{c}\text { Formulación } \\
\text { comercial }\end{array}$ \\
\hline bensulfurón-metil & Londax $60 \%$ GD \\
\hline pyrazosulfurón-etil & Sirius $10 \% \mathrm{PM}$ \\
\hline (Nombre clave) & Hoe 404 \\
\hline triclopir & Garlón 4 E \\
\hline bentazón + MCPA & Basagrán M-60 \\
\hline $2,4-\mathrm{D}$ & Rimaxil 720 SC \\
\hline 2,4-D + MCPA & Fenoxal \\
\hline ioxinil + 2,4-D & Actril DS \\
\hline
\end{tabular}

" La mención de nombre comerciales no indica recomendación o preferencia.

La aplicación de los herbicidas se hizo con un equipo de mochila accionado a presión constante por $\mathrm{CO}_{2}$, con boquillas 8002 , el volumen de aplicación fue de 400 litros por hectárea.

Durante las aplicaciones, los días fueron soleados, con viento calmo y el suelo estuvo saturado.

Para el control de poáceas se aplicó fenoxaprop-etil $90 \mathrm{~g} / \mathrm{ha}$, cuando la Echinocloa colona, que fue la poáceas dominante, tenía de dós a cuatro hojas y el arroz 2 a 3 hojas.

El diseño experimental usado fue de bloques completos al azar con cuatro repeticiones. Cada unidad experimental constó de $4 \mathrm{~m}$ de ancho y $6 \mathrm{~m}$ de largo.

Antes de hacer las aplicaciones se contó el número de ciperáceas por especie/m2 en el área experimental.

Las variables evaluadas fueron:
1. Síntomas de toxicidad en el cultivo durante los siguientes 20 días de la aplicación.

2. Número de hijos por planta $\left(\mathrm{n}=0,25 \mathrm{~m}^{2}\right)$

3. Número de espigas en $6 \mathrm{~m}^{2}$

4. Número de espigas por planta

4. Rendimiento, $\mathrm{N}=6 \mathrm{~m}^{2}$.

5. Porcentaje de control de C. iria a los 15, 30 y 60 días después de la aplicación.

6. Porcentaje de cobertura de ciperáceas a los 15,30 y 60 dda.

\section{RESULTADOS Y DISCUSIÓN}

\section{Efecto de los herbicidas sobre el Cyperus iria}

La población de C. iria en el lote donde se realizó este experimento fue uniforme y alcanzó cobertura total del arroz en el tratamiento testigo.

Los herbicidas que resultaron más eficaces para controlar esta maleza fueron las sulfonilureas, bensulfurónmetil y pyrazosulfuron-etil, que, durante todo el ciclo de la maleza, permitieron porcentajes de cobertura inferiores al $10 \%$ y control superior al $95 \%$, (Cuadro 3). Aunque ambos herbicidas mostraron excelente efecto sobre esta ciperácea, el pyrazosulfurón fue más rápido en causar toxicidad y muerte al Sontol, ya a los nueve días después de aplicado la mayoría del Sontol estaba muerto, razón por la que en la evaluación hecha 15 días después de la aplicación respectiva, fue el tratamiento que mostró el menor número de plantas vivas, (Cuadro 4). La lenta aparición de síntomas de toxicidad y muerte de las malezas se ha observado en varios herbicidas de este grupo, debido a que ellos inhiben la acción de la enzima Acetil Lactato Sintetasa, necesaria para la síntesis de los aminoácidos valina e isoleucina, sin embargo, se menciona que efectos sobre la división celular pueden ocurrir unas seis horas después de la aplicación, razón por la que aún cuando no se vean síntomas externos, el proceso de competencia se ve reducido desde un inicio, (Du Pont, s.f; Soto y Valverde, 1991). 
Cuadro 3. Porcentaje de control y cobertura de $C$. iria a los 15,30 y 60 días después de la aplicación de dos herbicidas. Hda. El Pelón, Liberia, Gte, 1993.

\begin{tabular}{|c|c|c|c|c|c|c|}
\hline \multirow{2}{*}{$\begin{array}{l}\text { Tratamiento } \\
\text { g i.a/ha }\end{array}$} & \multicolumn{3}{|c|}{$\%$ control } & \multicolumn{3}{|c|}{$\%$ cobertura } \\
\hline & 15 & 30 & 60 & 15 & 30 & 60 \\
\hline bensulfurón 48 & $96 a^{1 \prime}$ & $94 \mathrm{ab}$ & $100 \mathrm{a}$ & $1 \mathrm{~b}$ & $4 \mathrm{de}$ & 10 \\
\hline pirazulfuron 20 & $99 \mathrm{a}$ & $99 a$ & $99 a$ & $0 \mathrm{~b}$ & $0 \quad \mathrm{e}$ & 11 \\
\hline Hoe 40460 de PC & $72 \mathrm{a}$ & $66 \mathrm{c}$ & 72ab & $28 \mathrm{~b}$ & $24 \mathrm{~cd}$ & 4 \\
\hline triclopir 144 & $0 \mathrm{~b}$ & $0 \mathrm{~d}$ & $0 \mathrm{c}$ & $85 a$ & $98 \mathrm{a}$ & 18 \\
\hline triclopir 192 & $21 b$ & $11 \mathrm{~d}$ & $41 \mathrm{bc}$ & $72 \mathrm{a}$ & $75 \mathrm{~b}$ & 17 \\
\hline bentazón 720 & $67 a$ & $75 \mathrm{abc}$ & $21 \mathrm{c}$ & $26 \mathrm{~b}$ & 19 cde & 33 \\
\hline 2,4-D 500 & $79 \mathrm{a}$ & $74 \mathrm{bc}$ & $44 \mathrm{bc}$ & $29 \mathrm{~b}$ & 20 cde & 23 \\
\hline 2,4-D + MCPA 480 & $71 \mathrm{a}$ & $59 \mathrm{c}$ & $92 \mathrm{a}$ & $26 \mathrm{~b}$ & $34 \mathrm{c}$ & 6 \\
\hline ioxinil 100+2,4-D 600 & $86 a$ & $83 a b c$ & $44 \mathrm{bc}$ & $11 \mathrm{~b}$ & 12 cde & 5 \\
\hline Testigo con C. iria & - & - & - & 93 a & $99 a$ & 19 \\
\hline
\end{tabular}

"Promedios con igual letra en una misma columna presentan diferencias no significativas entre sí, según prueba de Tukey al $5 \%$. Los promedios corresponden a datos reales.

Cuadro 4. Efecto de los herbicidas sobre el número de ciperáceas. Liberia, Guanacaste, 1993.

\begin{tabular}{lcc}
\hline Tratamientos & $\mathbf{1 5} \mathbf{~ d d a}$ & $\mathbf{3 0 ~ d d a}$ \\
\hline bensulfurón 48 & $57 \mathrm{bc}$ & $37 \mathrm{~b}$ \\
pirazulfuron 20 & $0 \mathrm{c}$ & $8 \mathrm{~b}$ \\
Hoe 404 60 de PC & $284 \mathrm{ab}$ & $129 \mathrm{~b}$ \\
triclopir 144 & $205 \mathrm{abc}$ & $620 \mathrm{a}$ \\
triclopir 192 & $381 \mathrm{a}$ & $154 \mathrm{~b}$ \\
bentazón 720 & $164 \mathrm{abc}$ & $115 \mathrm{~b}$ \\
2,4-D 500 & $141 \mathrm{abc}$ & $121 \mathrm{~b}$ \\
2,4-D 480+ MCPA & $236 \mathrm{abc}$ & $96 \mathrm{~b}$ \\
ioxinil 100+2,4-D 600 & $143 \mathrm{abc}$ & $102 \mathrm{~b}$ \\
Testigo con C. iria & $154 \mathrm{abc}$ & $552 \mathrm{a}$ \\
\hline
\end{tabular}

"Promedios con igual letra en una misma columna presentan diferencias no significativas entre sí, según prueba de Tukey al 5\%. Los promedios corresponden a datos reales.

La alta eficacia del bensulfurón y pirazosulfurón en el control de $C$. iria encontrada en este experimento, coincide con observaciones hechas en parcelas satélites ubicadas en la cercanía del experimento, en las cuales se aplicó mezclas de benzulfurón o pyrazosulfurón con setoxidim, y en aplicaciones semicomerciales en la mis- ma finca con benzulfurón en lámina de agua. Estos resultados también concuerdan con la información publicada sobre la eficacia de las sulfonilureas en el combate de ciperáceas (Du Pont, s.f; WWSA, 1989).

Con respecto a los herbicidas de uso recomendado para el control de ciperáceas en arroz, se observó que mostraron. porcentajes intermedios en el control del Sontol; a excepción del triclopir que tuvo una pobre eficacia sobre esta maleza, aún en su dosis. alta (192 g i.a/ha). De ellos, la mezcla ioxinil + 2,4-D fue la más eficaz para controlar el Sontol, durante los siguientes 30 días después de aplicado (Cuadro 3). El control parcial de $C$. iria coincide con varias observaciones de campo y en parte explica el aumento gradual de estamaleza en las plantaciones de arroz con riego o anegado. En este sentido en varias observaciones de campo que se hicieron en los alrededores, se observó que $C$. iria fue más abundante en áreas saturadas o con láminas bajas, que en áreas con láminas más altas (15- $20 \mathrm{cms})$.

El C. iria concluyó su ciclo a los 70 días de edad, razón por la que la evaluación 60 días después de la aplicación de los herbicidas, no resultó significativa para porcentaje de cobertura. 
Esta observación también nos indica que la costumbre de algunos agricultores de hacer aplicaciones tardías, cuando el Sontol este en plena floración, no es efectiva, puesto que la mayor parte de la competencia ya ha ocurrido y el Sontol, aún sin herbicidas, morirá unos 20 días después.

En todos los casos hubo nueva germinación de esta maleza pero no alcanzó un grado importante de cobertura sobre el cultivo.

Fimbristylis sp. también se presentó en el experimento pero en menor cantidad, maduro aproximadamente a los 90 días.

\section{Efecto de los tratamientos sobre otras malezas}

De acuerdo a los objetivos propuestos y debido a que los herbicidas evaluados han mostrado eficacia en el control de ciperáceas y dicotiledóneas, pero no contra poáceas, (Soto y Agiiero, 1992; García y Fernández-Quintanilla, 1991; WWSA, 1989; Du Pont, s.f.; Irat, 1978); se decidió excluir la interferencia de las poáceas, mediante la. aplicación de fenoxaprop-etil, herbicida específico para este tipo de malezas y selectivo al arroz en aplicaciones de posemergencia cuando éste se encuentra entre las cuatro hojas y macollamiento completo (Hoechst, s.f.; WWSA, 1989; Soto y Agiiero, 1992). El control general de poáceas fue excelente, a excepción del tratamiento 2,4-D + MCPA, donde hubo presencia de Ischaemun rugosum, debido a que este esta situación fue igual en las cuatro repeticiones, se cree que pudo haber cierta interferencia de este tratamiento sobre el fenoxaprop, ya que la diferencia de aplicación entre ellos fue de un día; se conoce de incompatibilidad de este herbicida con hormonales cuando se aplican en mezcla (Hoechst, s.f.).

La presencia de malezas de hoja ancha fue relativamente baja, lo que sumado a la acción de los herbicidas, hizo que este grupo de malezas no interfiriera con los resultados obtenidos.

\section{Efecto de los herbicidas en el arroz}

Con respecto al efecto de los herbicidas en el arroz, unicamente se observó una muy leve clorosis en la última hoja del arroz en los tratamientos con sulfonilureas, posteriormente el arroz se recuperó. No se observaron diferencias significativas entre tratamientos, en el número de plantas, número de hijos $/ \mathrm{m}^{2}$ y número de hijos /planta. Pero sí se determinaron diferencias altamente significativas en el número de espigas y peso de grano por unidad de área. Nuevamente el pyrazosulfurón-etil y el bensulfurón-metil permitieron la mayor producción de arroz, en tanto en los tratamientos testigo y triclopir $0,144 \mathrm{~kg} / \mathrm{ha}$, donde hubo mayor cobertura de C. iria, se obtuvo la menor producción de arroz, (Cuadro 5), esto nos indica que C. iria redujo la producción de arroz en aproximadamene $50 \%$ cuando no se controló.

Cuadro 5. Efecto de los herbicidas en el número de espigas y peso de grano al $14 \%$ de humedad en $6 \mathrm{~m}^{2}$. Hda. El Pelón, Liberia, Gte.

\begin{tabular}{lcl}
\hline Tratamientos & No de espigas & Peso de grano \\
\hline bensulfurón 48 & $1631 \mathrm{ab}$ & $2304 \mathrm{ab}$ \\
pirazulfuron 20 & $2075 \mathrm{a}$ & $2594 \mathrm{a}$ \\
Hoe 404 60 de PC & $1634 \mathrm{ab}$ & $1800 \mathrm{bcd}$ \\
triclopir 144 & $1436 \mathrm{ab}$ & $1281 \mathrm{~d}$ \\
triclopir 192 & $1231 \mathrm{~b}$ & $1732 \mathrm{bcd}$ \\
bentazón 720 & $1585 \mathrm{ab}$ & $1910 \mathrm{abcd}$ \\
2,4-D 500 & $1479 \mathrm{ab}$ & $2119 \mathrm{abc}$ \\
2,4-D 480+ MCPA & $1299 \mathrm{~b}$ & $1354 \mathrm{~cd}$ \\
ioxinil 100+2,4-D 600 & $1659 \mathrm{ab}$ & $2069 \mathrm{abcd}$ \\
Testigo con C.iria & $1131 \mathrm{~b}$ & $1300 \mathrm{~d}$ \\
\hline
\end{tabular}

" Promedios con igual letra en una misma columna presentan diferencias no significativas entre sí, según prueba de Tukey al $5 \%$. Los promedios del\# de espigas corresponden a datos reales (no transformados)

Se pudo apreciar que altas poblaciones de C.iria pueden crecer vigorosamente durante los primeros 30 días después de la siembra del arroz, y llegar a cubrirlo totalmente. Después de la muerte del C. iria (ciclo 70 días) el arroz aparentemente se recupera, pero los daños, como en este caso, pueden alcanzar hasta un 50\% de reducción en rendimiento, pese al control del resto de malezas.

La práctica de aplicar algunos herbicidas hormonales cuando el $C$. iria esta en floración, no parece ser una bue- 
na alternativa, ya que en ese momento la mayor parte del daño ya ha sido hecho al arroz.

\section{CONCLUSIONES}

1. Los herbicidas más efectivos para el control de $C$. iria, fueron las sulfonilureas, bensulfurón-etil y pyrazosulfurón-metil, asu vez, fueron los tratamientos donde se obtuvo el mayor rendimiento del arroz.

2. Los herbicidas que tradicionalmente se usan para el control de ciperáceas, mostraron porcentajes intermedios de control de C. iria.

3. C. iria, aproximadamente a los 30 días después de la siembra, logró cubrir totalmente las plantas de arroz y redujo el rendimiento del arroz en granza en un 50\%.

4. C. iria completó su ciclo de vida en 70 días.

\section{LITERATURA CITADA}

ALPIZAR, P.E; BARRANTES, C.U. 1994. Comparación ecológica de las comunidades de malezas asociadas al arroz irrigado bajo dos sistemas de cultivo. In XL Reunión Anual del PCCMCA del 13 al 19 de marzo de 1994, San José, Costa Rica. Resúmenes. № 06.

DU PONT. s.f. Londax: herbicida. S.n.t. Bogotá, Colombia. 30 p.
GARCIA, T.L; FERNANDEZ, C. 1991. Fundamentos sobre malas hierbas y herbicidas. Ed. Mundi Prensa, Madrid, España. 348 p.

HOECHST. s.f. Furore: graminicida. S.n.t. Colombia. 20 p.

HOECHST. s.f. Veinticuatro respuestas sobre el manejo técnico de Furore. S.n.t. Colombia. 20 p.

HOLM, L.; PLUCKNETT, D.; PANCHO, J.; HERBERGER, J. 1977. The world's worst weeds, distribution and biology. Ed. The University Press of Hawaii, Hawaii. 609 p.

IRAT, RESEARCH CENTER GERDAT. 1978. Weeding of paddy-fields in West Africa and catalogue of the main weeds. Montpellier, Francia. 94 p.

SOTO, A. A; VALVERDE, B. 1991. Los herbicidas: propiedades fisicoquímicas, clasificación y mecanismos de acción. Ed. Universidad de Costa Rica. San José Costa Rica. 79 p.

SOTO, A A.; AGUERO, R. 1992. Combate químico de malezas en el cultivo del arroz. Ed. Universidad de Costa Rica. San José, Costa Rica. 81 p.

YAGUE, G.A.; TYLKO, B.I. 1992. Guía práctica de herbicidas. Ed. Maralpa. España. 264 p.

WEED SCIENCE SOCIETY OF AMERICA 1989. Herbicide handbook. Sixth edition. IIlinois, USA. 301 p. 